\title{
Influencia de la adición de galio en las propiedades estructurales y texturales de óxidos de $\mathrm{Mn}$ y Al
}

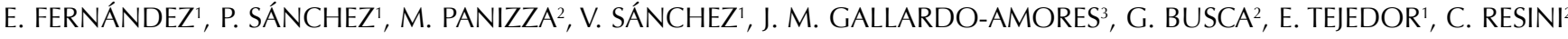 \\ 'Departamento de Química Inorgánica, $F^{a}$ de Ciencias Químicas, Universidad de Salamanca, 37008-Salamanca. \\ ${ }^{2}$ Dipartimento di Ingegneria Chimica e di Processo, I-16129 Genova (Italy). \\ ${ }^{3}$ Laboratorio Complutense de Altas Presiones, Departamento de Química Inorgánica I, U. Complutense, Ciudad Universitaria, E-28040 Madrid
}

\begin{abstract}
Se han obtenido óxidos mixtos de los sistemas Mn-Ga y Al-Ga en todo el intervalo de composiciones mediante un método de coprecipitación convencional y posteriores tratamientos térmicos. En el sistema Al-Ga, se observan soluciones sólidas hasta el $50 \%$ de Ga ${ }^{3+}$ identificándose la fase $\gamma-\mathrm{Al}_{2} \mathrm{O}_{3}$ por debajo de $800{ }^{\circ} \mathrm{C}$ y la fase $\alpha-\mathrm{Al}_{2} \mathrm{O}_{3}$ para temperaturas superiores. Las superficies específicas BET disminuyen con el incremento del contenido de Ga. La adición de $\mathrm{Ga}\left(10 \%\right.$ en moles y $\left.400{ }^{\circ} \mathrm{C}\right)$ al óxido de manganeso origina la obtención de fases termodinámicamente inestables, como el $\mathrm{MnO}_{2}$ (pirolusita), interesantes desde el punto de vista catalítico. El aumento de temperatura conduce a mezclas de fases $\mathrm{Mn}_{3} \mathrm{O}_{4}$ (hausmannita) y $\mathrm{Mn}_{2} \mathrm{O}_{3}$ (bixbyita) para contenidos de Ga mayores del 20\%. Estos datos se complementan por las variaciones observadas en los espectros FT-IR y electrónicos.

Palabras clave.- óxido de galio, óxido de manganeso, solución sólida, FT-IR.

\section{Influence of the $\mathrm{Ga}^{3+}$ doping in the textural and structural properties of the $\mathrm{Mn}$ and $\mathrm{Al}$ oxides}

$\mathrm{Mn}-\mathrm{Ga}$ and Al-Ga mixed oxides have been prepared in all compositional range by a conventional co precipitation method and subsequent thermal treatments. In the $\mathrm{Al}-\mathrm{Ga}$ mixed oxide system, solid solutions are observed for samples up to $50 \% \mathrm{Ga}$ content, identifying only the $\gamma-\mathrm{Al}_{2} \mathrm{O}_{3}$ phase below $800^{\circ} \mathrm{C}$ and the $\gamma-\mathrm{Al}_{2} \mathrm{O}_{3}$ phase for temperatures above $800{ }^{\circ} \mathrm{C}$. BET specific surface decreases significantly as Ga addition increases. Ga-addition to manganese oxide produce phases no thermodynamically stable, but very interesting from the catalytic point of view, i.e. $\mathrm{MnO}_{2}$ in samples with $10 \%$ Ga content and treated at $400{ }^{\circ} \mathrm{C}$. Increasing the temperature, biphasic materials composed by $\mathrm{Mn}_{3} \mathrm{O}_{4}$ (hausmannita) y $\mathrm{Mn}_{2} \mathrm{O}_{3}$ (bixbyita) phases are identified in the X-ray patterns for Ga contents $>20 \%$. These data are complemented by the evolution of FT-IR and electronic spectra.
\end{abstract}

Keywords.- gallium oxide, manganese oxide, solid solution, FT-IR

\section{INTRODUCCIÓN.}

El manganeso es un elemento que presenta un sistema de óxidos complejos entre los que destacan las siguientes formulaciones: $\mathrm{MnO}$, $\mathrm{Mn}_{3} \mathrm{O}_{4^{\prime}}, \mathrm{Mn}_{2} \mathrm{O}_{3}$ y $\mathrm{MnO}_{2}$ (1). Esta variabilidad de valencia ha atraído el interés en los últimos años para utilizarlo como fase activa de distintos catalizadores (Mn-Fe (2), Mn-Al (3)y Mn-Zr (4)) preparados por distintos métodos. Por otra parte, el óxido de aluminio en sus variedades, $\gamma$ y $\alpha$, es muy utilizado como soporte de fases activas y dopado convenientemente, se aprovecha su acidez superficial en procesos catalíticos. Además, el galio sirve para generar sitios ácidos Brönsted en estructuras abiertas como las zeolitas intercambiándolo por el $\mathrm{Al}$ (5). Estos materiales encuentran aplicaciones en catálisis heterogénea en la eliminación de compuestos orgánicos volátiles (6). Teniendo en cuenta estas bases, se emprendió el estudio de los sistemas de óxidos mixtos Mn-Ga y Al-Ga con objeto de ver las posibles sinergias que generaba este catión. En el presente trabajo, se presentan algunos de los resultados preliminares obtenidos.

\section{EXPERIMENTAL.}

Se prepararon diferentes muestras de los sistemas de óxidos mixtos de $\mathrm{Mn}_{\mathrm{x}} \mathrm{Ga}_{1-\mathrm{x}} \mathrm{O}_{\mathrm{y}}(\mathrm{x}=1,0.9,0.8,0.5,0.2$ y 0$)$ y de $\left(\mathrm{Al}_{\mathrm{x}} \mathrm{Ga}_{1-\mathrm{x}}\right)_{2} \mathrm{O}_{3}(\mathrm{x}=0$, $0.25,0.50,0.75$ y 1$)$ por un método de coprecipitación convencional descrito en previos trabajos (2). Los tratamientos térmicos posteriores de diferentes porciones fueron de 373, 723, 873, 1073 y $1223 \mathrm{~K}$ para el sistema Mn-Ga y de 373, 673, 1073 y 1473 K para el sistema Al-Ga. Las muestras se caracterizaron por técnicas convencionales de estado sólido: Difrac- ción de rayos $\mathrm{X}$ de polvo (DRX), espectroscopías FT-IR, Raman y RDUV-Vis, análisis TG-ATD y medidas de superficie específica.

\section{RESULTADOS}

\subsection{Sistema Mn-Ga}

Los parámetros cristalográficos de los precipitados y de las muestras calcinadas así como las fases identificadas mediante DRX se enumeran en la Tabla I. Los precipitados puros de Mn y Ga son materiales monofásicos formados por $\mathrm{Mn}_{3} \mathrm{O}_{4}$ (hausmannita) y $\beta-\mathrm{GaOOH}$, respectivamente. La adición de Ga da lugar a la aparición de las fase $\mathrm{HMnO}_{2}$ (groutita) y $\mathrm{MnOOH}$ (manganita) hasta composiciones por debajo del $50 \%$ de Ga, observándose posteriormente una solución sólida de oxohidróxidos tipo $\beta$ - $(\mathrm{Mn}, \mathrm{Ga}) \mathrm{OOH}$, donde el volumen y los parámetros de celda no varían sustancialmente debido a la similitud de los radios catiónicos de ambos metales. Por lo que respecta a los espectros FT-IR (no mostrados aquí), son consistentes con los aportados en la literatura para las muestras puras y en los óxidos mixtos se produce un desplazamiento notable hacia las bandas típicas de la fase $\beta-\mathrm{GaOOH}$ con el aumento del contenido $\mathrm{Ga}$, ya observado en las soluciones sólidas totales del sistema de óxidos mixtos de Fe-Ga (7). Por lo que se refiere a los espectros electrónicos, presentan una gran disminución de la intensidad con el contenido de Ga, debido al aislamiento de las especies de Mn que tienen transiciones $\mathrm{d} \square \mathrm{d}$. 
TABLA I.- PARÁMETROS CRISTALOGRÁFICOS DE LAS MUESTRAS DEL SISTEMA $\mathrm{Mn}_{\mathrm{x}} \mathrm{Ga}_{1-\mathrm{x}} \mathrm{O}_{\mathrm{y}}$.

\begin{tabular}{|c|c|c|c|c|c|c|}
\hline \multirow{2}{*}{ Muestra } & \multirow{2}{*}{$\mathbf{T}(\mathrm{K})$} & \multirow{2}{*}{ Fases } & \multicolumn{3}{|c|}{ Parámetros celda $(\AA ̊)$} & \multirow{2}{*}{$\mathrm{V}\left(\AA^{3}\right)$} \\
\hline & & & $\mathbf{a}$ & $\mathbf{b}$ & $\mathrm{c}$ & \\
\hline$x=1$ & \multirow{6}{*}{373} & $\mathrm{Mn}_{3} \mathrm{O}_{4}$ & 5.748 & & 9.440 & 312 \\
\hline$x=0.9$ & & $\begin{array}{c}\text { Groutita } \\
\text { Manganita }\end{array}$ & 4.505 & 10.29 & 2.891 & 134 \\
\hline$x=0.8$ & & $\begin{array}{c}\text { Groutita } \\
\text { Manganita }\end{array}$ & 4.519 & 10.23 & 2.898 & 134 \\
\hline$x=0.5$ & & $\beta-\mathrm{GaOOH}$ & 4.519 & 10.07 & 2.914 & 133 \\
\hline$x=0.2$ & & $\beta-\mathrm{GaOOH}$ & 4.513 & 9.835 & 2.942 & 131 \\
\hline$x=0$ & & $\beta-\mathrm{GaOOH}$ & 4.519 & 9.810 & 2.973 & 132 \\
\hline$x=1$ & \multirow{6}{*}{723} & $\mathrm{Mn}_{3} \mathrm{O}_{4}$ & 5.755 & & 9.459 & 313 \\
\hline$x=0.9$ & & $\mathrm{MnO}_{2}$ & 4.371 & & 2.860 & 54.8 \\
\hline $\mathrm{x}=0.8$ & & $\begin{array}{c}\mathrm{Mn}_{3} \mathrm{O}_{4} \\
\delta-\mathrm{Ga}_{2} \mathrm{O}_{3}\end{array}$ & $\begin{array}{l}5.553 \\
9.971 \\
\end{array}$ & & 9.472 & $\begin{array}{l}292 \\
991\end{array}$ \\
\hline$x=0.5$ & & $\delta-\mathrm{Ga}_{2} \mathrm{O}_{3}$ & 9.978 & & & 994 \\
\hline$x=0.2$ & & $\delta-\mathrm{Ga}_{2} \mathrm{O}_{3}$ & 9.986 & & & 996 \\
\hline$x=0$ & & $\alpha-\mathrm{Ga}_{2} \mathrm{O}_{3}$ & 4.979 & & 13.43 & 288 \\
\hline$x=1$ & \multirow{6}{*}{873} & $\mathrm{Mn}_{2} \mathrm{O}_{3}$ & 9.406 & 8.809 & 9.779 & 810 \\
\hline$x=0.9$ & & $\begin{array}{c}\mathrm{Mn}_{3} \mathrm{O}_{4} \\
\beta-\mathrm{Ga}_{2} \mathrm{O}_{3}\end{array}$ & $\begin{array}{l}5.990 \\
12.24 \\
\end{array}$ & $\begin{array}{l}2.951 \\
(102)\end{array}$ & $\begin{array}{l}9.473 \\
5.743 \\
\end{array}$ & $\begin{array}{l}340 \\
202 \\
\end{array}$ \\
\hline$x=0.8$ & & $\begin{array}{c}\mathrm{Mn}_{3} \mathrm{O}_{4} \\
\beta-\mathrm{Ga}_{2} \mathrm{O}_{3}\end{array}$ & $\begin{array}{l}5.937 \\
12.15 \\
\end{array}$ & $\begin{array}{l}2.960 \\
(102)\end{array}$ & $\begin{array}{l}9.551 \\
5.683 \\
\end{array}$ & $\begin{array}{l}337 \\
200 \\
\end{array}$ \\
\hline$x=0.5$ & & $\begin{array}{l}\beta-\mathrm{Ga}_{2} \mathrm{O}_{3} \\
\mathrm{Mn}_{3} \mathrm{O}_{4}\end{array}$ & 12.35 & $\begin{array}{l}2.977 \\
(104)\end{array}$ & 5.862 & 209 \\
\hline$x=0.2$ & & $\begin{array}{c}\beta-\mathrm{Ga}_{2} \mathrm{O}_{3} \\
\mathrm{Mn}_{3} \mathrm{O}_{4}\end{array}$ & 12.36 & $\begin{array}{c}2.962 \\
(10)\end{array}$ & 5.949 & 211 \\
\hline$x=0$ & & $\beta-\mathrm{Ga}_{2} \mathrm{O}_{3}$ & 12.38 & $\begin{array}{l}2.968 \\
(104)\end{array}$ & 6.017 & 214 \\
\hline$x=1$ & \multirow{6}{*}{1073} & $\mathrm{Mn}_{2} \mathrm{O}_{3}$ & 9.364 & 9.348 & 9.352 & 818 \\
\hline$x=0.9$ & & $\mathrm{Mn}_{2} \mathrm{O}_{3}$ & 9.399 & 9.387 & 9.382 & 828 \\
\hline$x=0.8$ & & $\begin{array}{l}\mathrm{Mn}_{3} \mathrm{O}_{4} \\
\mathrm{Mn}_{2} \mathrm{O}_{3}\end{array}$ & $\begin{array}{l}5.788 \\
9.388 \\
\end{array}$ & 9.368 & $\begin{array}{l}9.372 \\
9.377 \\
\end{array}$ & $\begin{array}{l}314 \\
825 \\
\end{array}$ \\
\hline$x=0.5$ & & $\begin{array}{c}\mathrm{Mn}_{2} \mathrm{O}_{3} \\
\beta-\mathrm{Ga}_{2} \mathrm{O}_{3} \\
\mathrm{MnGa}_{2} \mathrm{O}_{4}\end{array}$ & $\begin{array}{l}9.373 \\
12.14 \\
\\
8.281\end{array}$ & $\begin{array}{l}9.408 \\
3.009 \\
(107)\end{array}$ & $\begin{array}{l}9.443 \\
5.693\end{array}$ & $\begin{array}{l}833 \\
198 \\
568 \\
\end{array}$ \\
\hline$x=0.2$ & & $\beta-\mathrm{Ga}_{2} \mathrm{O}_{3}$ & 12.22 & $\begin{array}{l}3.045 \\
(104) \\
\end{array}$ & 5.812 & 210 \\
\hline$x=0$ & & $\beta-\mathrm{Ga}_{2} \mathrm{O}_{3}$ & 12.26 & $\begin{array}{l}3.033 \\
(104)\end{array}$ & 5.808 & 210 \\
\hline
\end{tabular}

El incremento de la temperatura hasta $723 \mathrm{~K}$ conduce a la estabilización del $\mathrm{MnO}_{2}$ (pirolusita) en óxidos mixtos con contenidos de $\mathrm{Ga}<10 \%$, hecho muy significativo y que sólo puede explicarse por la presencia en la estructura del catión $\mathrm{Ga}^{3+}$. Para composiciones entre el $10-50 \%$ de $\mathrm{Ga}$, se identifican mezclas de fases de $\mathrm{Mn}_{3} \mathrm{O}_{4}$ y $\delta-\mathrm{Ga}_{2} \mathrm{O}_{3}$. Este polimorfo del óxido de Ga de simetría cúbica se observa también para mayores contenidos, presentando de nuevo una solución sólida tipo $\delta$ - $(\mathrm{Ga}, \mathrm{Mn})_{2} \mathrm{O}_{3}$ para contenidos de $\mathrm{Ga}>50 \%$. Por lo que se refiere a los espectros FT-IR (Figura 1), la muestra pura de Mn muestra las características típicas de la fase $\mathrm{Mn}_{3} \mathrm{O}_{4}$ a 415, 510 y $615 \mathrm{~cm}^{-1}$. Estas bandas se van ensanchando progresivamente con el aumento del contenido de Ga hasta obtener las bandas típicas de la fase $\alpha-\mathrm{Ga}_{2} \mathrm{O}_{3}$; los óxidos mixtos muestran bandas adicionales que sugieren la presencia de una estructura $\alpha$-corindón distorsionada relacionada con la sustitución de Mn por Ga en la estructura. Mayores incrementos de temperatura dan lugar a la transición de fase $\alpha \square \beta$ del óxido de Ga, manteniendo soluciones sólidas tipo $\alpha-(\mathrm{Mn}, \mathrm{Ga})_{2} \mathrm{O}_{3}$ para contenidos de $\mathrm{Ga}<20 \%$ y tipo $\beta$ - $(\mathrm{Ga}, \mathrm{Mn})_{2} \mathrm{O}_{3}$ para contenidos de $\mathrm{Ga}>80 \%$.

\subsection{Sistema Al-Ga}

Los parámetros estructurales de los precitados junto con aquellos de las muestras calcinadas y las fases identificadas del sistema Al-Ga se muestran en la Tabla II. En general, los precipitados y las muestras calcinadas a temperaturas moderadas se caracterizan por ser materiales con baja cristalinidad, donde se detectan las fases $\alpha-\mathrm{GaOOH}$ y $\alpha-\mathrm{Ga}_{2} \mathrm{O}_{3}$ para altos contenidos de $\mathrm{Ga}$. A temperaturas de $473 \mathrm{~K}$ y superiores se detecta la fase $\gamma-\mathrm{Al}_{2} \mathrm{O}_{3}$ hasta contenidos de $\mathrm{Ga}<50 \%$. Incrementando la temperatura hasta $1073 \mathrm{~K}$, se produce la transición $\alpha \square \beta$ del óxido de Ga y se observan mezclas de fases para contenidos de $\mathrm{Ga}>50 \%$. Estos datos se ven corroborados por los espectros FT-IR y electrónicos (Figura 2). En la figura 2, se comparan los espectros electrónicos de las muestras calcinadas a $1073 \mathrm{~K}$, donde se observa la banda asignada a la transferencia de carga $\mathrm{O}^{2-} \rightarrow \mathrm{Al}^{3+}$ típica de espinelas defectivas, $\gamma-\mathrm{Al}_{2} \mathrm{O}_{3}$.

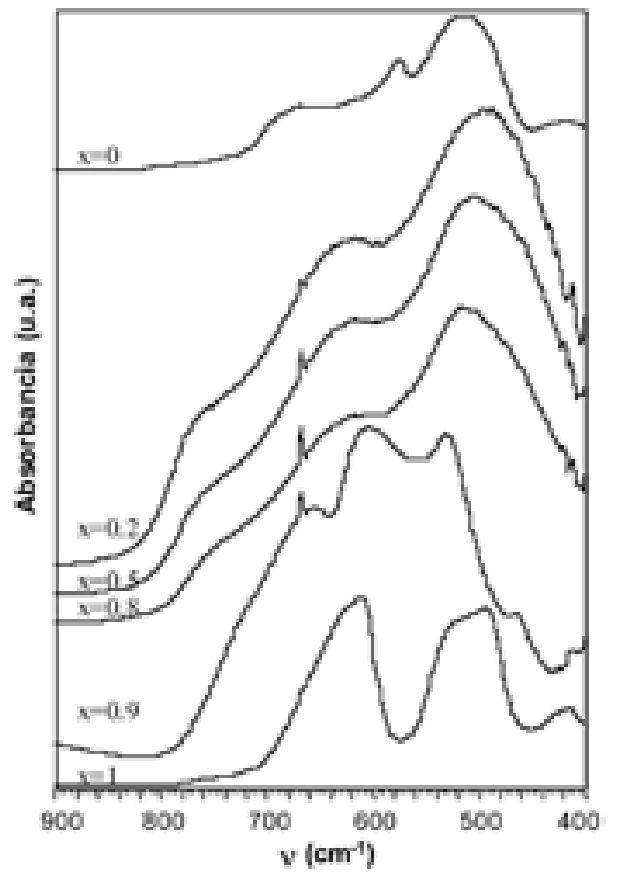

Figura 1. Espectros FT-IR de las muestras $\mathrm{Mn}_{x} \mathrm{Ga}_{1-\mathrm{x}} \mathrm{O}_{\mathrm{y}}$ a $723 \mathrm{~K}$

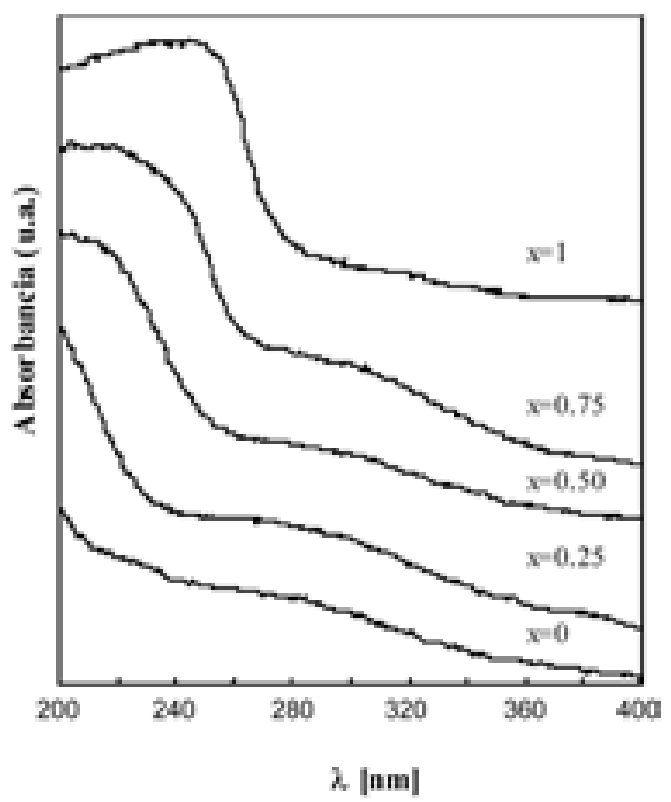

Figura 2. Espectros RD-VIS-UV de las muestras $\left(\mathrm{Al}_{x} \mathrm{Ga}_{1-\mathrm{x}}\right)_{2} \mathrm{O}_{3}$ a $1073 \mathrm{~K}$ 
Con el aumento del contenido de $\mathrm{Ga}^{3+}$ se produce una disminución de la intensidad y el desplazamiento del máximo hacia menores longitudes de onda relacionadas con transferencias de cargas del $\mathrm{O}^{2}-\mathrm{GG}^{3+}$ en un entorno octaédrico distorsionado.

TABLA II. PARÁMETROS CRISTALOGRÁFICOS DE LAS MUESTRAS DEL SISTEMA $\left(\mathrm{Al}_{\mathrm{x}} \mathrm{Ga}_{1-\mathrm{x}}\right)_{2} \mathrm{O}_{3}$

\begin{tabular}{|c|c|c|c|c|c|c|}
\hline \multirow{2}{*}{ Muestra } & \multirow{2}{*}{$\begin{array}{c}\mathrm{T} \\
(\mathrm{K})\end{array}$} & \multirow{2}{*}{ Fases } & \multicolumn{3}{|c|}{ Parámetros celda ( $(\AA)$} & \multirow[t]{2}{*}{$V\left(\AA^{3}\right)$} \\
\hline & & & $\mathbf{a}$ & b & c & \\
\hline$x=1$ & \multirow{5}{*}{373} & $\mathrm{NH}_{4} \mathrm{NO}_{3}$ & & & & \\
\hline$x=0.75$ & & $\mathrm{NH}_{4} \mathrm{NO}_{3}$ & & & & \\
\hline$x=0.50$ & & $\mathrm{NH}_{4} \mathrm{NO}_{3}$ & & & & \\
\hline$x=0.25$ & & $\begin{array}{l}\mathrm{NH}_{4} \mathrm{NO}_{3} \\
\mathrm{GaOOH}\end{array}$ & 4.549 & 9.755 & 2.953 & 131 \\
\hline$x=0$ & & $\mathrm{GaOOH}$ & 4.569 & 9.811 & 2.973 & 133 \\
\hline$x=1$ & \multirow{5}{*}{673} & $\gamma-\mathrm{Al}_{2} \mathrm{O}_{3}$ & & & & \\
\hline$x=0.75$ & & $\gamma-\mathrm{Al}_{2} \mathrm{O}_{3}$ & & & & \\
\hline$x=0.50$ & & $\gamma-\mathrm{Al}_{2} \mathrm{O}_{3}$ & & & & \\
\hline$x=0.25$ & & $\alpha-\mathrm{Ga}_{2} \mathrm{O}_{3}$ & 4.957 & & 13.37 & 284 \\
\hline$x=0$ & & $\alpha-\mathrm{Ga}_{2} \mathrm{O}_{3}$ & 4.979 & & 13.43 & 288 \\
\hline$x=1$ & \multirow{5}{*}{1073} & $\gamma-\mathrm{Al}_{2} \mathrm{O}_{3}$ & & & & \\
\hline$x=0.75$ & & $\gamma-\mathrm{Al}_{2} \mathrm{O}_{3}$ & & & & \\
\hline$x=0.50$ & & $\gamma-\mathrm{Al}_{2} \mathrm{O}_{3}$ & & & & \\
\hline$x=0.25$ & & $\begin{array}{l}\beta-\mathrm{Ga}_{2} \mathrm{O}_{3} \\
\alpha-\mathrm{Ga}_{2} \mathrm{O}_{3}\end{array}$ & 12.19 & $\begin{array}{l}3.020 \\
(104)\end{array}$ & 5.789 & 207 \\
\hline$x=0$ & & $\beta-\mathrm{Ga}_{2} \mathrm{O}_{3}$ & 12.26 & \begin{tabular}{|l}
3.033 \\
$(104)$
\end{tabular} & 5.808 & 210 \\
\hline$x=1$ & \multirow{5}{*}{1473} & $\alpha-\mathrm{Al}_{2} \mathrm{O}_{3}$ & 4.756 & & 12.97 & 254 \\
\hline$x=0.75$ & & $\begin{array}{l}\alpha-\mathrm{Al}_{2} \mathrm{O}_{3} \\
\beta-\mathrm{Ga}_{2} \mathrm{O}_{3}\end{array}$ & 4.786 & & 13.04 & 259 \\
\hline$x=0.50$ & & $\beta-\mathrm{Ga}_{2} \mathrm{O}_{3}$ & 11.59 & $\begin{array}{l}2.989 \\
(106)\end{array}$ & 5.885 & 196 \\
\hline$x=0.25$ & & $\beta-\mathrm{Ga}_{2} \mathrm{O}_{3}$ & 12.01 & $\begin{array}{c}3.001 \\
(103.78)\end{array}$ & 5.729 & 201 \\
\hline$x=0$ & & $\beta-\mathrm{Ga}_{2} \mathrm{O}_{3}$ & 12.19 & $\begin{array}{l}3.044 \\
(104)\end{array}$ & 5.789 & 209 \\
\hline
\end{tabular}

TABLA III. CARACTERÍSTICAS MORFOLÓGICAS DE LAS MUESTRAS DEL SISTEMA $\left(\mathrm{Al}_{\mathrm{x}} \mathrm{Ga}_{1-\mathrm{x}}\right)_{2} \mathrm{O}_{3}$

\begin{tabular}{|c|c|c|c|c|}
\hline Muestra & $\mathrm{T}[\mathrm{K}]$ & $\begin{array}{l}S_{\text {BET }} \\
{\left[\mathrm{m}^{2} / g\right]}\end{array}$ & $\begin{array}{l}\mathrm{V}_{\text {poro }} \\
{[\mathrm{ml} / \mathrm{g}]}\end{array}$ & $\begin{array}{c}\text { Diámetro medio } \\
\text { poro }[\mathrm{nm}]\end{array}$ \\
\hline$x=1$ & \multirow{5}{*}{673} & 227 & 0.14 & 1.68 \\
\hline$x=0.75$ & & 157 & 0.08 & 0.63 \\
\hline$x=0.50$ & & 167 & 0.06 & 1.08 \\
\hline$x=0.25$ & & 171 & 0.09 & 1.60 \\
\hline$x=0$ & & 50 & 0.11 & 1.56 \\
\hline$x=1$ & \multirow{5}{*}{1073} & 147 & 0.19 & 2.09 \\
\hline$x=0.75$ & & 98 & 0.07 & 1.61 \\
\hline$x=0.50$ & & 108 & 0.11 & 1.61 \\
\hline$x=0.25$ & & 64 & 0.07 & 1.71 \\
\hline$x=0$ & & 24 & 0.03 & 2.05 \\
\hline
\end{tabular}

\section{CONCLUSIONES}

1.- A temperaturas moderadas el Ga es un elemento muy efectivo en la estabilización de óxidos e hidróxidos de Mn que no son estables termodinámicamente.

2.- A altas concentraciones de Ga se tiende a favorecer las fases isoestructurales, mientras que a bajas concentraciones se favorecen fases con valencia reducida $\left(\mathrm{MnO}_{2}\right)$, de difícil estabilización incluso a temperaturas moderadas.

3.- A temperaturas moderadas los óxidos e hidróxidos mixtos de Mn-Ga presentan una solubilidad recíproca, mientras que a altas temperaturas ésta parece depender de la simetría del óxido de Ga (fase $\beta$ ). La solución sólida modifica sustancialmente los espectros electrónicos.

4.- En el sistema Al-Ga, el galio modifica notablemente las propiedades texturales.

5.- A temperaturas inferiores a $800{ }^{\circ} \mathrm{C}$ se forman soluciones sólidas con estructura $\gamma-\mathrm{Al}_{2} \mathrm{O}_{3}$ hasta el $50 \%$ de contenido en $\mathrm{Ga}$. $\mathrm{Al}$ aumentar la temperatura se produce la transición $\gamma \square \alpha \sin$ la segregación del $\mathrm{Ga}_{2} \mathrm{O}_{3}$.

\section{AGRADECIMIENTOS}

Los autores agradecen al MCYT (España) por la financiación mediante el proyecto (Mat. 2000-1153) y al INFM (Génova, Italia) por su apoyo. También se agradece la colaboración de D. Leonardo Hernández Delgado SALA-DIESEL (España). E. F. L., M. P. y V.S.E. agradecen a la JCYL (España), a la U. Génova y al MECD (España, Pr-2002-0164), respectivamente, por la concesión de becas de investigación.

\section{BIBLIOGRAFÍA}

1. A.M. Potter, G.R. Rossmann. «The tetravalent manganese oxides: identification, hydration, and structural relationships by infrared spectroscopy». Am. Mineralogist 64, 1199-1200 (1979).

2.. M. Baldi, V. S. Escribano, J. M. Gallardo, F. Milella, G. Busca. «Characterization of manganese and iron oxides as combustion catalysts for propane and propene». Appl. Catal. B, Environ. 17, L175-L182 (1998).

3.. E. Finocchio, G. Busca. «Characterization and hydrocarbon oxidation activity of coprecipitated mixed oxides $\mathrm{Mn}_{3} \mathrm{O}_{4} / \mathrm{Al}_{2} \mathrm{O}_{3}$ ». Catal. Today $70,213-225$ (2001).

4.. E. Fernández, V. S. Escribano, C. Resini, J. M. Gallardo, G. Busca. «A study of coprecipitated $\mathrm{Mn}-\mathrm{Zr}$ oxides and their behaviour as oxidation catalysts». Appl. Catal. B, Environ. 29, 251-261 (2001).

5.. K. Shimizu, A. Satsuma and T. Hattori, «Selective catalytic reduction of NO by hydrocarbons on $\mathrm{Ga}_{2} \mathrm{O}_{3} / \mathrm{Al}_{2} \mathrm{O}_{3}$ catalysts». App. Catal. B: Environ. 16, 319326 (1998).

6.. M. Baldi, E. Finocchio, F. Milella, G. Busca. «Catalytic combustion of C3 hydrocarbons and oxygenates over $\mathrm{Mn}_{3} \mathrm{O}_{4}{ }^{\Perp}$. Appl. Catal. B, Environ. $1643-$ 53 (1998).

7. J.M. Gallardo-Amores, V.S. Escribano and G. Busca. «Synthesis and characterization of Fe-Ga mixed hydroxide powders». J. Mater. Chem. 9, 1161-1168 (1999).

Recibido: 1.2 .03

Aceptado: 30.11 .03 